\title{
СХОДИМОСТЬ ТРИГОНОМЕТРИЧЕСКИХ РЯДОВ ФУРЬЕ ФУНКЦИЙ С ОГРАНИЧЕНИЕМ НА ФРАКТАЛЬНОСТЬ ИХ ГРАФИКОВ ${ }^{1}$
}

\section{М. Л. Гриднев}

Для непрерывной на отрезке функции $f$ вводится понятие модуля фрактальности $\nu(f, \varepsilon)$ как функции, которая каждому $\varepsilon>0$ сопоставляет минимальное число квадратов размера $\varepsilon$, которыми можно покрыть график функции $f$. В терминах модуля фрактальности и модуля непрерывности $\omega(f, \delta)$ получено условие равномерной сходимости ряда Фурье функции $f$ : если

$$
\omega(f, \pi / n) \ln \left(\frac{\nu(f, \pi / n)}{n}\right) \longrightarrow 0 \quad \text { при } n \longrightarrow+\infty
$$

то ряд Фурье функции $f$ сходится равномерно. Это условие уточняет известный признак сходимости Дини-Липшица. Кроме того, получена равномерная по $x \in[0,2 \pi]$ оценка порядка роста сумм Фурье $S_{n}(f, x)$ непрерывной функции $f$ :

$$
S_{n}(f, x)=o\left(\ln \left(\frac{\nu(f, \pi / n)}{n}\right)\right)
$$

Показано, что эта оценка является неулучшаемой.

Ключевые слова: тригонометрический ряд Фурье, равномерная сходимость, фрактальная размерность.

M. L. Gridnev. Convergence of trigonometric Fourier series of functions with a constraint on the fractality of their graphs.

For a function $f$ continuous on a closed interval, its modulus of fractality $\nu(f, \varepsilon)$ is defined as the function that maps any $\varepsilon>0$ to the smallest number of squares of size $\varepsilon$ that cover the graph of $f$. The following condition for the uniform convergence of the Fourier series of $f$ is obtained in terms of the modulus of fractality and the modulus of continuity $\omega(f, \delta)$ : if

$$
\omega(f, \pi / n) \ln \left(\frac{\nu(f, \pi / n)}{n}\right) \longrightarrow 0 \text { as } n \longrightarrow+\infty
$$

then the Fourier series of $f$ converges uniformly. This condition refines the known Dini-Lipschitz test. In addition, for the growth order of the partial sums $S_{n}(f, x)$ of a continuous function $f$, we derive an estimate that is uniform in $x \in[0,2 \pi]$ :

$$
S_{n}(f, x)=o\left(\ln \left(\frac{\nu(f, \pi / n)}{n}\right)\right)
$$

The optimality of this estimate is shown.

Keywords: trigonometric Fourier series, uniform convergence, fractal dimension.

MSC: $42 \mathrm{~A} 20$

DOI: $10.21538 / 0134-4889-2018-24-4-104-109$

Пусть $f-2 \pi$-периодическая суммируемая на $[0,2 \pi]$ функция. Напомним, что ее коэффициенты и частные суммы Фурье определяются следующим образом:

$$
a_{k}=\frac{1}{\pi} \int_{-\pi}^{\pi} f(t) \cos k t d t, \quad b_{k}=\frac{1}{\pi} \int_{-\pi}^{\pi} f(t) \sin k t d t, \quad S_{n}(f, x)=\frac{a_{0}}{2}+\sum_{k=1}^{n}\left(a_{k} \cos k x+b_{k} \sin k x\right) .
$$

\footnotetext{
${ }^{1}$ Исследование выполнено за счет гранта Российского научного фонда (проект 14-11-00702).
} 
Для функции $f$, определенной на множестве $X \subset \mathbb{R}$, через $\omega_{X}(f, \delta)$ обозначим ее модуль непрерывности

$$
\omega_{X}(f, \delta)=\sup \{|f(x)-f(y)|: x, y \in X,|x-y| \leqslant \delta\} .
$$

Через $C_{2 \pi}$ будем обозначать множество всех определенных на $\mathbb{R}$ непрерывных $2 \pi$-периодических функций.

В теории тригонометрических рядов интерес представляет вопрос об условиях сходимости рядов Фурье. Хорошо известен

Признак Дини-Липшица. Пусть $f \in C_{2 \pi}$ u ее модуль непреръвности $\omega(f, \delta)=$ $\omega_{\mathbb{R}}(f, \delta)$ удовлетворяет условию

$$
\omega(f, \delta) \ln \left(\frac{1}{\delta}\right) \longrightarrow 0 \quad \text { nрu } \delta \longrightarrow+0 .
$$

Тогда ряд Фурье функиии $f$ сходится равномерно на $[0,2 \pi]$.

Этот признак, как известно, является неулучшаемым. В данной статье мы получим уточнение этого признака для более узкого класса функций.

О п р е д е л е н и е 1 . Пусть дана ограниченная функция $f:[a, b] \rightarrow \mathbb{R}$. Модулем фрактальности функции $f$ на отрезке $[a, b]$ будем называть функцию $\nu_{[a, b]}(f, \varepsilon)$, которая любому $\varepsilon$, большему нуля, сопоставляет минимальное число замкнутых квадратов со сторонами длины $\varepsilon$, параллельными осям координат, которыми можно покрыть график функции $f$ на отрезке $[a, b]$.

3 а м е ч а н и е 1. Из определения модуля фрактальности следует, что

$$
\frac{b-a}{\varepsilon} \leqslant \nu_{[a, b]}(f, \varepsilon) \leqslant\left(\frac{b-a}{\varepsilon}+1\right)\left(\frac{\max \{|f(x)-f(y)|: x, y \in[a, b]\}}{\varepsilon}+1\right)=O\left(\frac{1}{\varepsilon^{2}}\right) .
$$

Понятие модуля фрактальности было предложено Н. Ю. Антоновым и С. В. Бердышевым и, насколько нам известно, в опубликованном виде встречалось лишь в работах автора настоящей статьи $[1 ; 2]$.

О п р е д е л е н и е 2 . Пусть $\mu:(0,+\infty) \rightarrow(0,+\infty)$ - невозрастающая функция. Определим функциональный класс $F^{\mu}$ следующим образом:

$$
F^{\mu}:=\left\{f \in C_{2 \pi}: \nu_{[0,2 \pi]}(f, \varepsilon)=O(\mu(\varepsilon)), \varepsilon>0\right\} .
$$

3 а м е ч а н и е 2. График любой функции из $F^{\mu}$, где $\mu(\varepsilon)=1 / \varepsilon^{\alpha}, \quad \alpha \in[1,2]$, имеет фрактальную размерность по Минковскому, не большую $\alpha$, что и обусловливает название модуля фрактальности.

Лемма 1. Если $f$-непрерывная на $[a, b]$ функция $u[\alpha, \beta] \subset[a, b]$, то

$$
\frac{\max \{|f(x)-f(y)|: x, y \in[\alpha, \beta]\}}{\beta-\alpha} \leqslant \nu_{[\alpha, \beta]}(f, \beta-\alpha) \leqslant \frac{\omega_{[a, b]}(f, \beta-\alpha)}{\beta-\alpha}+1 .
$$

Д о к а з а т е л ь с т в о следует из того наблюдения, что количество квадратов со стороной длины $\beta-\alpha$ в минимальном покрытии графика непрерывной функции $f$ на отрезке $[\alpha, \beta]$ равно округлению вверх числа $\max \{|f(x)-f(y)| /(\beta-\alpha): x, y \in[\alpha, \beta]\}$.

Обозначим отрезок $\left[\frac{(i-1) \pi}{n}, \frac{i \pi}{n}\right]$ как $I(n, i)$.

Лемма 2. Если $f$ - непрерывная на $[0,2 \pi]$ функиия, то

$$
\sum_{i=1}^{2 n} \nu_{I(n, i)}(f, \pi / n) \leqslant 3 \nu_{[0,2 \pi]}(f, \pi / n) .
$$


Д о к а з а т е л ь с т в о. Пусть $K-$ множество квадратов со стороной $\pi / n$, составляющих минимальное покрытие функции $f$ на отрезке $[0,2 \pi], \sharp K-$ мощность множества $K$. Согласно определению модуля фрактальности $\sharp K=\nu_{[0,2 \pi]}(f, \pi / n)$. Для $i \in\{1, \ldots, 2 n\}$ обозначим через $K_{i}$ подмножество множества $K$, состоящее из квадратов, имеющих непустое пересечение с полосой $I(n, i) \times \mathbb{R}$. Ясно, что объединение всех квадратов из $K_{i}$ покрывает участок графика функции $f$, соответствующий отрезку $I(n, i)$. Поскольку $\nu_{I(n, i)}(f, \pi / n)$ - минимальное число квадратов, которыми можно покрыть участок графика функции $f$, соответствующий отрезку $I(n, i)$, то $\nu_{I(n, i)}(f, \pi / n) \leq \sharp K_{i}$. Учитывая, что каждый квадрат из $K_{i}$ может пересекаться не более чем с тремя полосами вида $I(n, j) \times \mathbb{R}(j$ может быть равным $i-1, i$ или $i+1)$, получаем

$$
\sum_{i=1}^{2 n} \nu_{I(n, i)}(f, \pi / n) \leq \sum_{i=1}^{2 n} \sharp K_{i} \leq 3 \sharp K=3 \nu_{[0,2 \pi]}(f, \pi / n) .
$$

Лемма 2 доказана.

Докажем следующее вспомогательное утверждение.

Лемма 3. Пусть для набора неотрищательных чисел $\left\{a_{i}\right\}_{i=1}^{n}$ известны следующие ограничения: $\max _{1 \leqslant i \leqslant n} a_{i} \leqslant A$ u $\sum_{i=1}^{n} a_{i} \leqslant A B$ при некоторых $A$ и В. Тогда

$$
\sum_{i=1}^{n} \frac{a_{i}}{i} \leqslant 2 A \ln (B+1)
$$

Д о к а з а т е л ь с т в о. При $B \geqslant n$ утверждение очевидно. Если $0 \leqslant B<1$, то требуемое неравенство получается с помощью неравенства $B \leqslant 2 \ln (B+1)$, которое следует из разложения в ряд Тейлора величины $\ln (B+1)$. Рассмотрим случай $1 \leqslant B<n$. Обоснуем неравенство

$$
\sum_{i=1}^{n} \frac{a_{i}}{i} \leqslant \sum_{1 \leqslant i \leqslant B} \frac{A}{i}
$$

Действительно,

$$
\sum_{1 \leqslant i \leqslant B} \frac{A}{i}-\sum_{i=1}^{n} \frac{a_{i}}{i}=\sum_{1 \leqslant i \leqslant B} \frac{A-a_{i}}{i}-\sum_{B<i \leqslant n} \frac{a_{i}}{i} \geqslant \frac{1}{B}\left(\sum_{1 \leqslant i \leqslant B}\left(A-a_{i}\right)-\sum_{B<i \leqslant n} a_{i}\right)=\frac{1}{B}\left(A B-\sum_{i=1}^{n} a_{i}\right) \geqslant 0 .
$$

Теперь получим искомую оценку:

$$
\sum_{1 \leqslant i \leqslant B} \frac{A}{i} \leqslant A \sum_{1 \leqslant i \leqslant B} \int_{i}^{i+1} \frac{2}{t} d t \leqslant 2 A \int_{1}^{B+1} \frac{1}{t} d t=2 A \ln (B+1) .
$$

Лемма 3 доказана.

Теорема 1. Пусть $f$-непрерывная $2 \pi$-периодическая функиия. Тогда для всех $x \in[0,2 \pi]$

$$
\left|S_{n}(f, x)-f(x)\right| \leqslant C \omega(f, \pi / n) \ln \left(\frac{\nu_{[0,2 \pi]}(f, \pi / n)}{n}\right)+o(1)
$$

где $C$ - абсолютная константа и оценка о(1) равномерная по $x$ nри $n \rightarrow \infty$.

Д о к а з а т е л ь с т в о. Следуя доказательству признака Салема $[3$, гл. $4, \S 5]$, можно увидеть, что при нечетных $n$

$$
\left|S_{n}(f, x)-f(x)\right| \leqslant \frac{4}{\pi^{2}}\left(\max _{0 \leqslant t \leqslant 2 \pi}\left|T_{n}(t)\right|+\max _{0 \leqslant t \leqslant 2 \pi}\left|Q_{n}(t)\right|\right)+o(1),
$$


где

$$
T_{n}(x)=\frac{f(x)-f\left(x+\frac{\pi}{n}\right)}{1}+\frac{f\left(x+\frac{2 \pi}{n}\right)-f\left(x+\frac{3 \pi}{n}\right)}{3}+\ldots+\frac{f\left(x+\frac{(n-1) \pi}{n}\right)-f(x+\pi)}{n}
$$

и

$$
Q_{n}(x)=\frac{f(x)-f\left(x-\frac{\pi}{n}\right)}{1}+\frac{f\left(x-\frac{2 \pi}{n}\right)-f\left(x-\frac{3 \pi}{n}\right)}{3}+\ldots+\frac{f\left(x-\frac{(n-1) \pi}{n}\right)-f(x-\pi)}{n} .
$$

Оценим абсолютные значения $T_{n}(x)$ и $Q_{n}(x)$ сверху через выражения из правой части (3). Рассмотрим $T_{n}(x)$. Случай с $Q_{n}(x)$ рассматривается аналогично.

Применив левое неравенство из (1) и добавив недостающие члены к получившейся сумме, получаем

$$
\left|T_{n}(x)\right| \leqslant \sum_{i=1}^{n} \frac{\pi \nu_{\left[x+\frac{(i-1) \pi}{n}, x+\frac{i \pi}{n}\right]}(f, \pi / n)}{i n} .
$$

При любом фиксированном $x \in[0,2 \pi]$, каждому $i \in \mathbb{N}$ сопоставим минимальное целое $k_{i}$, для которого $\left[x+\frac{(i-1) \pi}{n}, x+\frac{i \pi}{n}\right] \subset I\left(n, k_{i}\right) \cup I\left(n, k_{i}+1\right)$ и соответственно

$$
\nu_{\left[x+\frac{(i-1) \pi}{n}, x+\frac{i \pi}{n}\right]}(f, \pi / n) \leqslant \nu_{I\left(n, k_{i}\right)}(f, \pi / n)+\nu_{I\left(n, k_{i}+1\right)}(f, \pi / n) .
$$

Тогда имеем

$$
\sum_{i=1}^{n} \frac{\pi \nu_{\left[x+\frac{(i-1) \pi}{n}, x+\frac{i \pi}{n}\right]}(f, \pi / n)}{i n} \leqslant \sum_{i=1}^{n} \frac{\pi \nu_{I\left(n, k_{i}\right)}(f, \pi / n)+\pi \nu_{I\left(n, k_{i}+1\right)}(f, \pi / n)}{i n} .
$$

Нетрудно видеть, что для каждого $k=1, \ldots, 2 n$ величина $\nu_{I(n, k)}(f, \pi / n)$ встречается в правой части (5) не более двух раз (в силу периодичности $f$, отрезки $I(n, i)$ и $I(n, i+2 k n)$ считаем одинаковыми). Используя этот факт и лемму 2 , получаем

$$
\sum_{i=1}^{n} \frac{\pi \nu_{I\left(n, k_{i}\right)}(f, \pi / n)+\pi \nu_{I\left(n, k_{i}+1\right)}(f, \pi / n)}{n} \leqslant \frac{2 \pi}{n} \sum_{k=1}^{2 n} \nu_{I(n, k)}(f, \pi / n) \leqslant \frac{6 \pi \nu_{[0,2 \pi]}(f, \pi / n)}{n} .
$$

Кроме того, согласно лемме 1 для всех $i \in\{1, \ldots, n\}$

$$
\frac{\pi \nu_{I\left(n, k_{i}\right)}(f, \pi / n)+\pi \nu_{I\left(n, k_{i}+1\right)}(f, \pi / n)}{n} \leqslant 2 \omega(f, \pi / n)+\frac{2 \pi}{n} .
$$

Теперь, имея (6), (7), воспользуемся леммой 3. Также пользуясь замечанием после определения 1 , получаем

$$
\begin{gathered}
\sum_{i=1}^{n} \frac{\pi \nu_{I\left(n, k_{i}\right)}(f, \pi / n)+\pi \nu_{I\left(n, k_{i}+1\right)}(f, \pi / n)}{i n} \leqslant 4\left(\omega(f, \pi / n)+\frac{\pi}{n}\right) \ln \left(\frac{3 \pi \nu_{[0,2 \pi]}(f, \pi / n)}{n \omega(f, \pi / n)+\pi}+1\right) \\
=4 \omega(f, \pi / n) \ln \left(\frac{3 \pi \nu_{[0,2 \pi]}(f, \pi / n)+n \omega(f, \pi / n)+\pi}{n}\right)+\frac{4 \pi}{n} \ln \left(\frac{3 \pi \nu_{[0,2 \pi]}(f, \pi / n)+n \omega(f, \pi / n)+\pi}{n}\right) \\
+4\left(\omega(f, \pi / n)+\frac{\pi}{n}\right) \ln \left(\frac{1}{\omega(f, \pi / n)+\pi / n}\right) \\
\leqslant 4 \omega(f, \pi / n) \ln \left(\frac{3 \pi \nu_{[0,2 \pi]}(f, \pi / n)}{n}+1\right)+\frac{4 \pi}{n} \ln (C n)+o(1) \leqslant 4 \omega(f, \pi / n) \ln \left(\frac{\nu_{[0,2 \pi]}^{5}(f, \pi / n)}{n^{5}}\right)+o(1) \\
\leqslant 20 \omega(f, \pi / n) \ln \left(\frac{\nu_{[0,2 \pi]}(f, \pi / n)}{n}\right)+o(1),
\end{gathered}
$$

что вместе с (4) и (5) завершает получение оценки (3) для нечетных $n$. Ясно, что тогда для четных $n$ оценка (3) также имеет место. Теорема доказана.

Из доказанной теоремы автоматически вытекают два следствия. 
Следствие 1. Пусть $f$-непрерывная $2 \pi$-периодическая функиия и ее модули непрерывности и фрактальности удовлетворяют условию

$$
\omega(f, \pi / n) \ln \left(\frac{\nu_{[0,2 \pi]}(f, \pi / n)}{n}\right) \longrightarrow 0 \text { npu } n \longrightarrow+\infty .
$$

Тогда ряд Фуръе функиии $f$ сходится равномерно на $[0,2 \pi]$.

Следствие 2. Пусть $f$-непрерывная $2 \pi$-периодическая функиия и $f \notin F^{1 / \varepsilon}$. Тогда равномерно по $x \in[0,2 \pi]$ имеет место оц,нка

$$
S_{n}(f, x)=o\left(\ln \left(\frac{\nu_{[0,2 \pi]}(f, \pi / n)}{n}\right)\right) .
$$

3 а м е ч а н и е 3 . Если в следствиях 1 и 2 функция $f$ принадлежит $F^{\mu}$, где $\mu(\varepsilon)=1 / \varepsilon^{\alpha}$, $1<\alpha \leqslant 2$, то мы получим признак Дини-Липшица и известную оценку для непрерывных функций $S_{n}(f, x)=o(\ln n)$ соответственно, которые, как известно, неулучшаемы. Если же $\mu(\varepsilon)$ - функция, стремящаяся к бесконечности в нуле медленнее, чем $1 / \varepsilon^{\alpha}$, при любом $\alpha>1$, но быстрее чем $1 / \varepsilon$, например, если $\mu(\varepsilon)=\ln (1 / \varepsilon) / \varepsilon$, то получим усиление данных утверждений для более узкого, чем $C_{2 \pi}$, класса функций $F^{\mu}$.

Следующее утверждение показывает, что оценка (8) является неулучшаемой.

Теорема 2. Пусть $\mu:(0,+\infty) \rightarrow(0,+\infty)$ - непрерьвная функиия, $\varepsilon \mu(\varepsilon)$ не возрастает и стремится $\kappa+\infty$ при $\varepsilon \rightarrow+0$. Тогда для любой неубывающей последовательности положительных чисел $\left\{\lambda_{n}\right\}_{n=1}^{\infty}$, удовлетворлющей условию

$$
\lambda_{n}=o\left(\ln \left(\frac{\mu(\pi / n)}{n}\right)\right),
$$

найдется функиия $f \in F^{\mu}$ такая, что

$$
\limsup _{n \rightarrow \infty} \frac{\left|S_{n}(f, 0)\right|}{\lambda_{n}}>0
$$

Д о к а з а т е л ь с т в о. Искомую функцию $f \in F^{\mu}$ заимствуем из доказательства теоремы 1 в [2], где единственным отличием будут другие коэффициенты $c_{k}$, участвующие в определении $f$. А именно, по определенным в той же статье последовательностям $a_{k}$ и $k_{i}$ коэффициенты $c_{k}$ теперь будут определяться следующим образом:

$$
c_{k}=\left\{\begin{array}{cc}
\lambda_{a_{k_{i}}} / \ln \left(\frac{3 \mu\left(\pi / a_{k_{i}}\right)}{a_{k_{i}}}-1\right), & k \in\left\{k_{i}\right\}_{i=1}^{\infty} ; \\
0, & k \notin\left\{k_{i}\right\}_{i=1}^{\infty} .
\end{array}\right.
$$

Тогда из конструкции $f$ будет вытекать

$$
S_{a_{k_{i}}}(f, 0) \geqslant \frac{c_{k_{i}}}{\pi} \ln \frac{a_{k_{i}}}{a_{k_{i}-1}}+o(1) \geqslant \frac{c_{k_{i}}}{\pi} \ln \left(3 \mu\left(\frac{\pi}{a_{k}}\right) \frac{1}{a_{k}}-1\right)+o(1)=\lambda_{a_{k_{i}}} / \pi+o(1),
$$

что и требовалось. Теорема доказана.

\section{СПИСОК ЛИТЕРАТУРЫ}

1. Гриднев М.Л. О классах функций с ограничением на фрактальность их графика // Proc. of the 48th Internat. Youth School-Conf. "Modern Problems in Mathematics and its Applications". Yekaterinburg, 2017. Vol. 1894. C. 167-173. URL: http://ceur-ws.org/Vol-1894/appr5.pdf. 
2. Gridnev M.L. Divergence of Fourier series of continuous functions with restriction on the fractality of their graphs // Ural Math. J. 2017. Vol. 3, no. 2. P 46-50. doi: 10.15826/umj.2017.2.007.

3. Бари Н.К. Тригонометрические ряды. М.: ГИМФЛ, 1961. 937 с.

Поступила 31.08.2018

После доработки 28.10.2018

Гриднев Максим Леонидович

Принята к публикации 05.11.2018

младший науч. сотрудник

Институт математики и механики им. Н.Н. Красовского УрО РАН,

г. Екатеринбург

e-mail: coraxcoraxg@gmail.com

\section{REFERENCES}

1. Gridnev M. L. On classes of functions with a restriction on the fractality of their graphs. In: A. A. Makhnev, S. F. Pravdin (eds.): Proc. of the 48th Internat. Youth School-Conf. "Modern Problems in Mathematics and its Applications", Yekaterinburg, 2017, vol. 1894, pp. 167-173 (in Russian). Published at http://ceur-ws.org/Vol-1894/appr5.pdf .

2. Gridnev M. L. Divergence of Fourier series of continuous functions with restriction on the fractality of their graphs. Ural Math. J., 2017, vol. 3, no. 2, pp. 46-50. doi: 10.15826/umj.2017.2.007.

3. Bary N.K. A treatise on trigonometric series, vol. I; II. Oxford; N Y: Pergamon Press, 1964, 553 p.; 508 p. doi: 10.1002/zamm.19650450531. Original Russian text published in Bari N.K. Trigonometricheskie ryady, Moscow: GIMFL Publ., 1961, 937 p.

Received August 31, 2018

Revised October 28, 2018

Accepted November 05, 2018

Funding Agency: This work was supported by the Russian Science Foundation (project no. 1411-00702).

Maksim Leonidovich Gridnev, Krasovskii Institute of Mathematics and Mechanics, Ural Branch of the Russian Academy of Sciences, Yekaterinburg, 620990 Russia, e-mail: coraxcoraxg@gmail.com . 